\title{
THE ROLE OF IONIZATION ELECTRONS IN DIRECT NEUTRALINO DETECTION
}

\author{
J.D. Vergados ${ }^{1 *}$ and H. Ejiri ${ }^{2}$ \\ 1 Physics Department, University of Cyprus, 1678 Nicosia, Cyprus \\ and \\ Theoretical Physics Division, T-6, LANL, P.O. Box 1663, Los Alamos, N.M. 87545, \\ $U S A$. \\ * 2 NS, International Christian University, Osawa, Mitaka, Tokyo, 181-8585, \\ Japan \\ and \\ Emeritus RCNP, Osaka University, Ibaraki, Osaka 567-0047,Japan
}

\begin{abstract}
In this paper we estimate the event rates of neutralino-nucleus scattering leading to electron emission by the atomic ionization. We find that the branching ratio for detecting electrons vis a vis the traditional neutralino nucleus elastic scattering sensitively depends on the threshold energy. In the case of a light target and a neutralino mass of $100 \mathrm{GeV}$ we estimate it to be around 10 percent assuming a reasonable threshold energy of $250 \mathrm{eV}$.
\end{abstract}

Key words: LSP, Direct neutralino search, ionization electrons, electron detection, dark matter,WIMP.

PACS numbers:95.+d, 12.60.Jv.

ऋ Permanent address: University of Ioannina, Gr 451 10, Ioannina, Greece. E-mail:Vergados@cc.uoi.gr 


\section{Introduction}

The combined MAXIMA-1 [1], BOOMERANG [2], DASI [3] and COBE/DMR [4] Cosmic Microwave Background (CMB) observations as well as the recent WMAP data [5] imply that the Universe is flat [6] and that most of the matter in the Universe is Dark, i.e. exotic. Crudely speaking one has:

$$
\Omega_{b}=0.05, \Omega_{C D M}=0.30, \Omega_{\Lambda}=0.65
$$

for the baryonic, dark matter and dark energy fractions respectively. Furthermore, since the non exotic component cannot exceed $40 \%$ of the CDM [8], there is room for the exotic WIMP (Weakly Interacting Massive Particles). Many experiments are currently under way aiming at the direct detection of WIMP. In fact the DAMA experiment [9] has claimed the observation of such events, which with better statistics have subsequently been interpreted as a modulation signal [10]. These data, however, are not consistent with other recent experiments, see e.g. EDELWEISS [11] and CDMS [12].

Supersymmetry naturally provides candidates for the dark matter constituents [13],[14]-[17]. In the most favored scenario of supersymmetry the lightest supersymmetric particle (LSP) can be simply described as a Majorana fermion, a linear combination of the neutral components of the gauginos and higgsinos [13],[14][24].

The event rates, however, are expected to be quite low and the nuclear recoil energies are extremely small. Thus one has to try to reduce the background to the lowest possible level and understand possible reaction induced backgrounds(for a brief discussion see the appendix at the end of the paper). Furthermore one has to search for characteristic signatures associated with this reaction. Examples are the modulation of the event rates with the motion of the Earth (modulation effect) and the correlation of the observed rates of directionally sensitive experiments with the 
motion of the sun $[25,26,27]$. Transitions to low energy excited nuclear states have also been considered [28,29].

In the present paper we will explore another novel possibility, namely detecting dark matter constituents by observing the low energy electrons, which follow the ionization of the atom during the LSP-nucleus collision. This possibility may be realized with the technology of gaseous TPC detectors [30]. In fact the WIMP-nucleus scattering leads: (i) The nuclear recoil and (ii) to nuclear recoil with atomic excitation. So far most CDM searches have been made by the inclusive processes, (i) and (ii), employing solid detectors. We propose that the produced electrons in atomic excitation (ii) should be studied by exclusive measurements.

\section{The effect of bound electrons}

The differential cross section for the LSP nucleus scattering leading to the emission of electrons in the case of non relativistic neutralinos takes the form:

$$
\begin{aligned}
d \sigma(\mathbf{k}) & =\frac{1}{v} \frac{m_{e}}{E_{e}}|M|^{2} \frac{d^{3} \mathbf{q}}{(2 \pi)^{3}} \frac{d^{3} \mathbf{k}}{(2 \pi)^{3}}(2 \pi)^{3} \frac{1}{2(2 \ell+1)} \\
\sum_{n \ell m} p_{n \ell} & {\left[\tilde{\phi}_{n \ell m}(\mathbf{k})\right]^{2} 2 \pi \delta\left(T_{\chi}+\epsilon_{n \ell}-T-\frac{q^{2}}{2 m_{A}}-\frac{\left(\mathbf{p}_{\chi}-\mathbf{k}-\mathbf{q}\right)^{2}}{2 m_{\chi}}\right), }
\end{aligned}
$$

where $v, T_{x}$ and $\mathbf{p}_{\chi}$ are the oncoming LSP velocity, energy and momentum respectively, while $\mathbf{q}$ is the momentum transferred to the nucleus. $M$ is the invariant amplitude, known from the standard neutralino nucleus cross section, $T$ and $\mathbf{k}$ are the kinetic energy and momentum of the outgoing electron and $\epsilon_{n \ell}$ is the binding energy of the initial electron. $\tilde{\phi}_{n \ell m}(\mathbf{k})$ is the Fourier transform of the bound electron wave function, i.e its wave function in momentum space. $p_{n \ell}$ is the probability of finding the electron in the $n, \ell$ orbit.

In the expression above and in what follows our normalization 
will consist of one electron per atom, to be compared with the cross section per nucleus of the standard experiments.

The binding energies for hydrogenic wave functions are given by:

$$
\epsilon_{n}=-\frac{m_{e}(Z \alpha)^{2}}{2 n^{2}}
$$

One, of course, may have to introduce effective charges, especially for heavy atoms. Some hydrogenic wave functions for light systems are going to be given below.

After summing over the m-substates we find that:

$$
\frac{1}{2(2 \ell+1)} \sum_{m}\left|\tilde{\phi}_{n \ell m}(\mathbf{k})\right|^{2}=\frac{1}{8 \pi}\left|\tilde{\phi}_{n \ell}(k)\right|^{2} .
$$

Due to the delta function the integration over the momentum $q$ is trivial and yields

$$
\int q^{2} d q \delta\left(T_{\chi}+\epsilon_{n \ell}-T-\frac{q^{2}}{2 m_{A}}-\frac{\left(\mathbf{p}_{\chi}-\mathbf{k}-\mathbf{q}\right)^{2}}{2 m_{\chi}}\right)=v K \mu_{r}^{2} \frac{(\xi+\Lambda)^{2}}{\Lambda},
$$

with $\mu_{r}$ the LSP-nucleus reduced mass and

$$
\Lambda=\sqrt{\xi^{2}+\frac{m_{\chi}}{\mu_{r}}\left(\frac{1}{K^{2}}-1\right)-\frac{m_{\chi}}{\mu_{r} K^{2}} \frac{\left(T-\epsilon_{n \ell}\right)}{T_{\chi}}},
$$

with

$$
\mathbf{K}=\frac{\mathbf{p}_{\chi}-\mathbf{k}}{p_{\chi}}, K=\frac{\sqrt{p_{\chi}^{2}+k^{2}-2 k p_{\chi} \xi_{1}}}{p_{\chi}}, \xi_{1}=\hat{p}_{\chi} \cdot \hat{k}, \xi=\hat{q} \cdot \hat{K}
$$

In order to avoid any complications arising from questions regarding the allowed SUSY parameter space, a subject extensively studied anyway [13]-[24], we will present our results normalized to the standard neutralino nucleus cross section. The thus obtained branching ratios are independent of all parameters of supersymmetry except the neutralino mass. The numerical results given here apply in the case of the coherent mode. If, however, we limit ourselves to the ratios of the relevant cross sections, we do not expect substantial changes in the case of the spin induced process. 
With these ingredients we find that the ratio of the cross section with ionization divided by that of the standard neutralinonucleus elastic scattering, nuclear recoil experiments (nrec), takes the form:

$$
\begin{aligned}
\frac{d \sigma(T)}{\sigma_{n r e c}}= & \frac{1}{4} \sum_{n \ell} p_{n \ell}\left|\tilde{\phi_{n \ell}}\left(2 m_{e} T\right)\right|^{2} \\
& \frac{\int_{-1}^{1} d \xi_{1} \int_{\xi_{L}}^{1} d \xi K \frac{(\xi+\Lambda)^{2}}{\Lambda}\left[F\left(\mu_{r} v(\xi+\Lambda)\right)\right]^{2}}{\int_{0}^{1} 2 \xi d \xi\left[F\left(2 \mu_{r} v \xi\right)\right]^{2}} m_{e} k d T,
\end{aligned}
$$

where $\xi_{L}=\sqrt{\frac{m_{\chi}}{\mu_{r}}\left[1+\frac{1}{K^{2}}\left(\frac{T-\epsilon_{n \ell}}{T_{\chi}}-1\right)\right]}, 2 \frac{\mu_{r}}{m_{\chi}} p_{\chi} \xi=2 \mu_{r} v \xi$ is the momentum $q$ transferred to the nucleus and $F(q)$ is the nuclear form factor. The outgoing electron energy lies in the range $0 \leq T \leq$ $\frac{\mu_{r}}{m_{\chi}} T_{\chi}-\epsilon_{n \ell}$. The expression for the standard cross section $\sigma_{n r e c}$ will be given below (Eq. 9).

Since the momentum of the outgoing electron is much smaller than the momentum of the oncoming neutralino, i.e. $K \approx 1$, the integration over $\xi_{1}$ can be trivially performed. Furthermore, if the effect of the nuclear form factor can be neglected, the integration over $\xi$ can be performed analytically. Thus we get:

$$
\begin{aligned}
\frac{d \sigma(T)}{\sigma_{n r e c}} & =\frac{1}{2} \sum_{n \ell} p_{n \ell}\left|\tilde{\phi_{n \ell}}\left(\sqrt{2 m_{e} T}\right)\right|^{2}\left[1-\left(\frac{m_{\chi}}{\mu_{r}} \frac{\left(T-\epsilon_{n \ell}\right)}{T_{\chi}}\right)\right. \\
& \left.+\sqrt{1-\left(\frac{m_{\chi}}{\mu_{r}} \frac{\left(T-\epsilon_{n \ell}\right)}{T_{\chi}}\right)}\right] m_{e} \sqrt{2 m_{e} T} d T .
\end{aligned}
$$

Otherwise the angular integrations can only be done numerically. Furthermore integrating numerically the above expression over the electron spectrum we obtain the total cross section. One, of course, must convolute the above expression with the velocity distribution (see next section) to obtain both the differential rate as well the total rate as a function of the neutralino mass.

Before proceeding further we will give an estimate of the expected standard elastic scattering recoil coherent events. The LSP- nu- 
cleus cross-section takes the form:

$$
\sigma_{\text {nrec }}=\left(\frac{\mu_{r}}{\mu_{r}(p)}\right)^{2} A^{2} \sigma_{p} \int_{0}^{1} 2 d \xi\left[F\left(2 \mu_{r} v \xi\right)\right]^{2},
$$

where $\sigma_{p}$ is the LSP-proton cross section and $\mu_{r}(p) \approx m_{p}$ is the reduced mass for the proton-LSP system. From this expression we see the coherent effect of all nucleons arising from the elementary scalar interaction. In the case of a light target like ${ }^{20} \mathrm{Ne}$ the effect of the form factor is not large. The event rate, corresponding to an LSP velocity $\sqrt{\left\langle v^{2}\right\rangle}$, takes the form:

$$
R=\frac{d N}{d t}=\frac{\rho(0)}{m_{\chi}} \frac{m}{A m_{p}} \sqrt{\left\langle v^{2}\right\rangle}\left(\frac{\mu_{r}}{\mu_{r}(p)}\right)^{2} A^{2} \sigma_{p},
$$

where $\rho(0)=0.3 \mathrm{GeV} / \mathrm{cm}^{3}$ is the LSP density in our vicinity and $\mathrm{m}$ is the detector mass. The upper limit of the current experiments is $\sigma_{p} \leq 10^{-5} \mathrm{pb}$. Using this limit with $m_{\chi}=100 \mathrm{GeV}$ and $\sqrt{\left\langle v^{2}\right\rangle}=270 \mathrm{~km} / \mathrm{s}$, we get for the $\mathrm{A}=20$ system

$$
\frac{d N}{d t} \approx 8 \text { events } /(y \cdot k g-\text { target }) \text {. }
$$

This rate will be become $\approx 10$ events/(y. $\mathrm{kg}$-target $)$, if the folding with the LSP velocity distribution is taken into account (see sec. 4).

\section{A brief discussion of the backgrounds}

We must begin by emphasizing that the standard dark matter experiments have thus far consisted of inclusive measurements employing solid detectors, where the electrons and the recoil nuclei have not been separated. These measure continuum energy spectra of the recoiling nuclei with an energy resolution, which is not very good. Most experiments are employing neutrons with energy in the range of $5-10 \mathrm{MeV}$ with a resolution of a few $0.1 \mathrm{MeV}$, which is much larger than the electron energy. Then the electron contribution is hidden. The present paper is concerned with an 
exclusive measurement, whereby the electron signal is separated from the nuclear recoil signal. The electron rate is about $10 \%$ of the nuclear recoil rate. The actual nuclear recoil rate is reduced by about $30-50 \%$ depending the detector ienergy cutoff. It thus appears that the electron rate could be as high as $1 / 3$ of the nuclear recoil rate. This makes it a realistic proposal, if the BG problems are under control. The nice feature of an exclusive experiment is that one can measure simultaneously the electrons and the nuclear recoils, substantially reducing this way the BG rate.

To circumvent the BG problems one must use high purity materials and run the experiment underground. The main source of background is neutrons and this applies both in the standard experiments as well as the present novel proposal. It thus appears that background events of primary concern to the present work can arise from two main sources:

(1) Reaction induced Auger electrons. These can arise from the decay of the inner electron holes in the daughter atom. The decay width has been estimated to be $1.2 \times 10^{-9} \mathrm{keV}$ for ${ }^{20} \mathrm{Ne}$. These electrons can have energies in the region of interest. In the case of ${ }^{20} \mathrm{Ne}$ they are expected at energies of $0.77,0.80$ and $0.83 \mathrm{keV}$ depending on which two electrons fill this hole and produce the Auger electron [30]. The reaction induced 1s holes, however, occur with a small probability, see Fig. 1 One may also have background electrons, produced from impurities which may lead to betas and photoelectrons producing $\gamma$ rays. Anyway it has been estimated that such a background is under control [30].

(2) Radioactive Impurities (RI). RI 's give rise to $\beta, \gamma, \alpha$ BG's. As it is well known, recent recoil detectors can separate the recoil signal from electron backgrounds of RI $\beta$, $\gamma$ 's. Similarly the electron tracking detectors select electron signals. It is quite realistic, as shown both in the recent solar $\nu$ detectors and the planned $\beta \beta$ experiments, to build detectors at the level of ppt of U-Th impurities $\left(\mathrm{ppt}=10^{-12}\right)$. Note that most RI BG's 
deposit $\beta, \gamma$ energies at multi-sites. Then the $\mathrm{BG}$ rate for the electron detectors with impurities at the level of a few ppt is estimated at 30 per year per ton, by a stringent selection of DM single-site signals from the RI multi-site events. This is orders of magnitude less than the present limit $(8 /$ year $/ \mathrm{kg}$ ) of the WIMP's. It should also be noted that this limit is 2-3 orders of magnitude less than the electron signal rates ( $1 \sim 10$ per year per ton) and the electron BG rates of the planned electron detectors for real time solar $\mathrm{p}-\mathrm{p} \nu$ 's and $\beta \beta$ experiments. In any case it seems feasible to build detectors with BG rates well below the the signal rates with $10^{-5} \mathrm{pb}$. Furthermore since the electron energy signal is always accompanied by the recoil signal, the coincidence measurement of both the electron tracks and and the recoil signals will make this experiment almost free from BG events.

(3) Electron backgrounds due to RI. These are under control [30]. Most electrons from RI's are $\beta$ rays followed by $\gamma$ rays and Compton electrons followed by Compton $\gamma$ rays. These deposit energy at multi-sites and, thus, they can be eliminated by selecting the single-site event from WIMP's. With a purity level of the order of $\mu \mathrm{Bq}$ per $\mathrm{Kg}$ one gets a $\mathrm{BG}$ event rate of less than 0.1 per year per $\mathrm{kg}$, after a stringent signal selection. This is far below the present WIMP signal rate ( 8 per y per $\mathrm{kg}$ ).

(4) Neutron induced events. This source is also present in the standard nuclear recoil experiments and it should be dealt with in both. Such neutron events can be deciphered by studying the reaction products. We will, however, estimate the event rate due to neutrons using the distribution for muon produced neutrons given at $2000 \mathrm{~m}$ w.e. by Gaitskell [31].

- Neutron electron scattering due to the neutron magnetic dipole moment.

This process is very special in the sense that the electron interacts directly with the neutron. Thus the magnetic moment interaction does not suffer from suppression at high electron energies due to the bound electron form factor, see 
Figs 4- 5 obtained as discussed in the appendix.

- Electron ionization due to neutron-nucleus elastic scattering.

This process is characterized by kinematics similar to the reaction we are interested in. For the model discussed in the appendix involving a Yukawa interaction, see Fig. 6, we obtain the results shown in Figs 7-8.

It appears that both of these backgrounds yield rates that could be troublesome. In practice the rate gets smaller by 2-3 orders of magnitude at typical underground labs with 4000 - $6000 \mathrm{~m}$ w.e. Active neutron shields for incoming and outgoing neutrons reduce further the BG rate. Accordingly the $B G$ rate due to the neutrons can be negligibly small in realistic cases. These BG problems are further discussed in the Appendix.

\section{Some results}

We will now apply the above formalism for a typical case, namely $m_{\chi}=100 \mathrm{GeV}$. We will employ a Maxwell-Boltzmann LSP velocity distribution with respect to the galactic center [27], namely:

$$
f(\mathbf{v})=\frac{1}{\left(v_{0} \sqrt{\pi}\right)^{3}} e^{-\left(v^{2} / v_{0}^{2}\right)},
$$

with $v_{0}=220 \mathrm{~km} / \mathrm{s}$. On this we impose by hand an upper velocity bound (escape velocity), $v_{e s c}=2.84 v_{0}$. We then transform this distribution to the lab frame, $\mathbf{v} \rightarrow \mathbf{v}+\mathbf{v}_{0}$, where $\mathbf{v}_{0}$ is the velocity of the sun around the center of the galaxy. We will not be concerned here with the motion of the the Earth around the sun, i.e. the modulation effect [27].

Folding both the numerator and the denominator of Eq. (7) with the LSP velocity distribution, after multiplying each with the LSP flux $\frac{\rho(0)}{m_{\chi}} \frac{m}{A m_{p}} v$, we obtain the differential ratio $\frac{1}{R} \frac{d R_{e}}{d T}$, with $R_{e}$ the rate for ionization. If we then integrate the final expression with respect to the electron energy, we obtain the ratio $\frac{R_{e}}{R}$. By 
doing the same in the case of Eq. (8), we obtain the analogous ratios $\frac{1}{R_{0}} \frac{d R_{e 0}}{d T}$ and $\frac{R_{e 0}}{R_{0}}$, associated with the case without nuclear form factor.

We will apply our results on a light target, e.g. ${ }^{19} \mathrm{~F}$, which is considered in the standard nuclear recoil experiments. The obtained results are essentially identical to those for ${ }^{20} \mathrm{Ne}$, which is a popular gaseous TPC counter currently being considered for detection of low energy neutrinos produced in triton decay. Our approach can, of course, also be applied in the case of the heavier targets, employed in current dark matter searches, but the atomic physics involved is much more complicated

The radial functions $\tilde{\phi_{n \ell}}(k)$ will be given in terms of a dimensionless variable $b=\frac{n k a_{B}}{2 z}$, by writing $\tilde{\phi_{n \ell}}(k) \Rightarrow R_{n \ell}(b)$, with $R_{n \ell}^{2}(b)$ given by:

$$
\begin{gathered}
R_{1 s}^{2}=\frac{256}{\left(1+4 b^{2}\right)^{4} \pi} \\
R_{2 s}^{2}=\frac{512\left(1-4 b^{2}\right)^{2}}{\left(1+4 b^{2}\right)^{4} \pi}, R_{2 p}^{2}=\frac{16384 b^{2}}{3\left(1+4 b^{2}\right)^{6} \pi} .
\end{gathered}
$$

The binding energies employed are given in the above order by [32]:

$$
\epsilon_{1 s}=-0.870, \epsilon_{2 s}=-0.048, \epsilon_{2 p}=-0.021 .
$$

In Fig. 1 we show the differential rate of our process, divided by the total nuclear recoil event rate, for each orbit (see Eq. 8) without the inclusion of the nuclear form factor on the left and with appropriate form factor [33] on the right. In Fig. 2 we show the same quantity taking all orbitals together. These results were obtained considering one electron per atom and weighing each orbit with the probabilities $p_{n \ell}$ :

$$
p_{n \ell}=(2 / 10,2 / 10,6 / 10) .
$$

The branching ratio for the total rate leading to our process, obtained as discussed above, are shown in Fig. 3, as a function of the low energy detector cut-off (threshold). From this plot we see that the introduction of the nuclear form factor, which we view 
as quite reliable [33], does not substantially alter the branching ratio. This trend for the branching ratio may persist even for heavier nuclei, even though the individual rates may depend on the assumed form factor.

From these plots we see that, even though the differential rate peaks at low energies, there remains substantial strength above the electron energy of $0.2 \mathrm{keV}$, which is the threshold energy used in MICROMEGAS detectors, like the one recently [30] proposed.

\section{Conclusions}

Based on our results summarized in the previous section one can be optimistic about using the emitted electrons in the neutralino nucleus collisions for the direct detection of the LSP. This novel process may be exploited by the planned TPC low energy electron detectors. By achieving low energy thresholds of about $0.25 \mathrm{keV}$, the branching ratios are approximately 10 percent. They can be even larger, if one includes low energy cutoffs imposed by the detectors in the standard experiments, not included in the above estimate.

As we have seen the background problems associated with the proposed mechanism are not worse than those entering the standard experiments. In any case coincidence experiments with xrays, produced following the de-excitation of the residual atom, may help reduce the background events to extremely low levels.

Acknowledgments: This work was supported in part by the European Union under the contracts RTN No HPRN-CT-2000-00148 and MRTN-CT-2004-503369. One of the authors (JDV) is indebted to Professors K. Zioutas for a careful reading of the manuscript and Y. Giomataris and N. Alexandropoulos for their useful comments regarding experimental issues related to this work. 

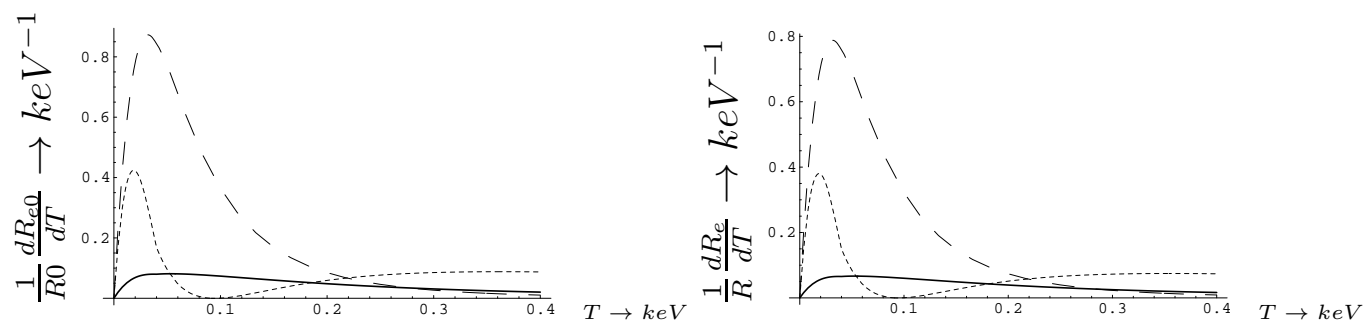

Fig. 1. The differential rate, divided by the total rate associated with the nuclear recoils, as a function of the electron energy $\mathrm{T}$ (in $\mathrm{keV}$ ). Each atomic orbit involved in the target ${ }^{20} \mathrm{Ne}$ is included separately. The results exhibited are obtained without nuclear form factor on the left and with nuclear form factor on the right. The full line, the short-dashed line and the long-dashed line correspond to the orbits $1 s, 2 s$ and $2 p$ respectively.
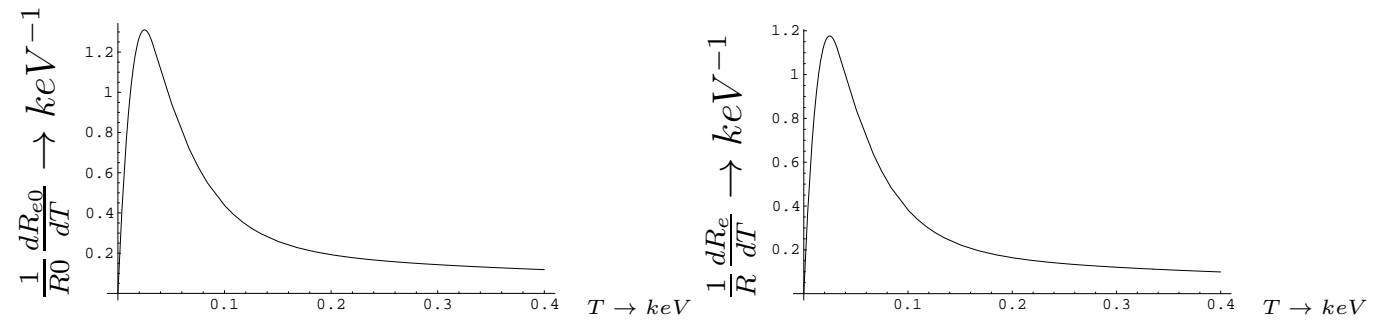

Fig. 2. The same as in Fig. 1 by summing the contribution of all orbitals together.

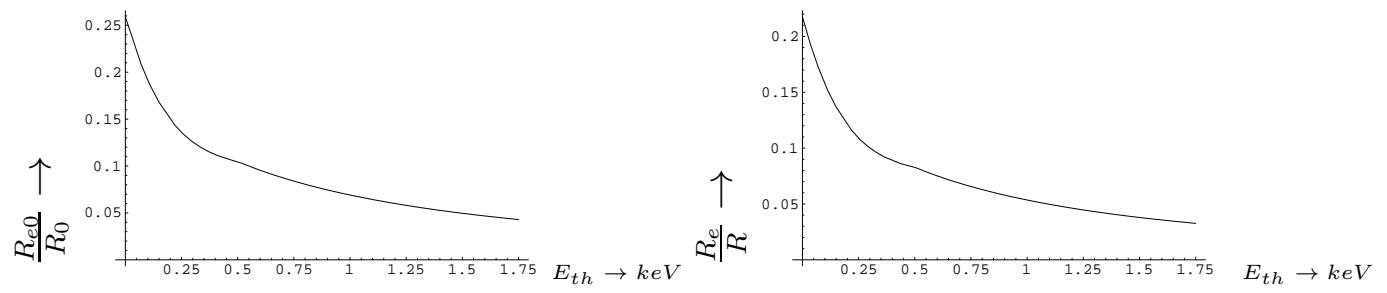

Fig. 3. The total rate for ionization divided by the standard nuclear recoil rate as a function of the threshold energy. No low energy cutoff imposed by the detector was included in the computation of the standard rate. The estimated rate for the proposed mechanism is obtained by multiplying this branching ratio with the standard event rate. In the case of ${ }^{20} \mathrm{Ne}$ this about 10 events/(kg-y), obtained from the upper bound of the cross section as derived from the current experiments.

Appendix: Some further discussion on the background.

In this section we are going to briefly discuss the possible background (BG) events. A more detailed discussion will be given elsewhere. RI impurities in all detector components should be as low as possible to avoid all kinds of BG electrons from $\beta$ and $\gamma$ rays from RI. In practice the Ur and Th impurities in the rock at underground laboratories can be reduced to be less than $10^{-16}$ in weight leading to a BG event rate of 0.03 per Kg per year, much 

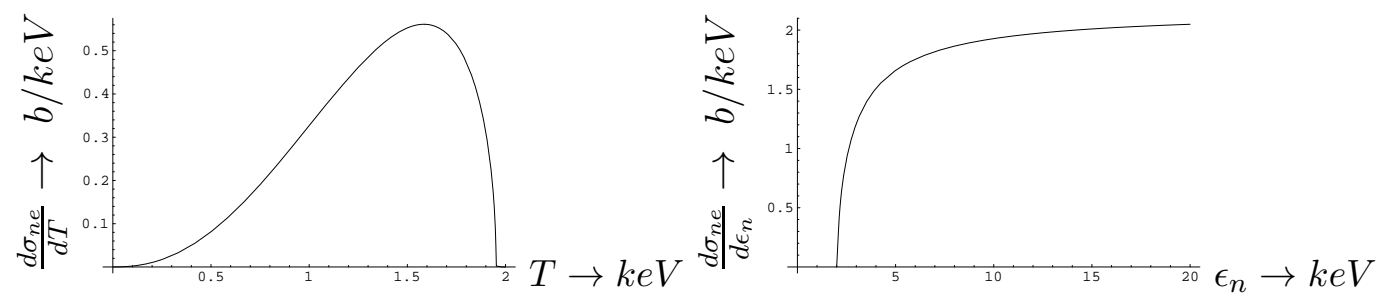

Fig. 4. The differential cross section for neutron electron scattering due to the neutron magnetic moment in the case ${ }^{20} \mathrm{Ne}$. The results on the left correspond to an incident neutron energy of $2 \mathrm{keV}$ plotted as a function of the outgoing electron energy for electrons bound in the $1 s, 2 s$ and $2 p$ orbitals. The results on the right correspond to an outgoing electron of $2 \mathrm{keV}$ as a function of the incident neutron energy.

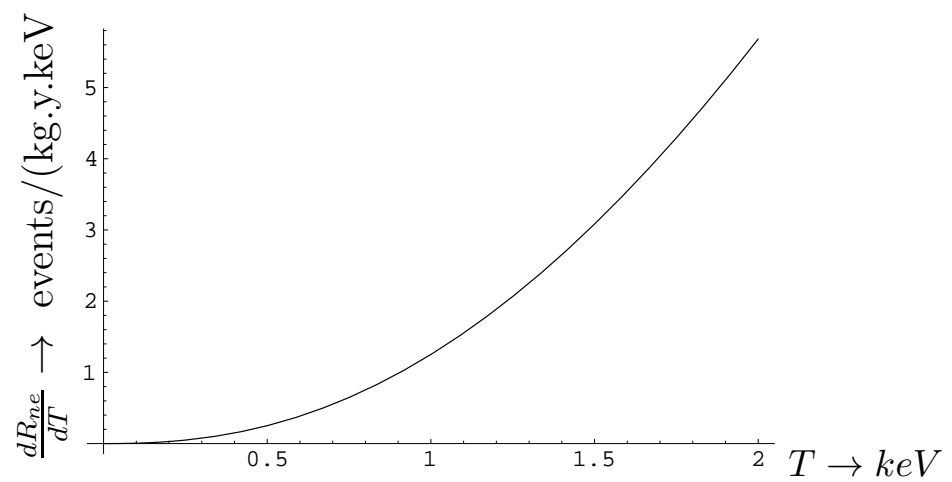

Fig. 5. The differential event rate for electron ionization in neutron electron scattering, which is due to the neutron magnetic moment, in the case ${ }^{20} \mathrm{Ne}$ as a function of the outgoing electron energy. Electrons bound in the $1 s, 2 s$ and $2 p$ orbitals were considered . The results presented were obtained using distribution of Gaitskell for muon produced neutrons. All neutrons were included, but the event rate reached saturation in about $500 \mathrm{MeV}$.

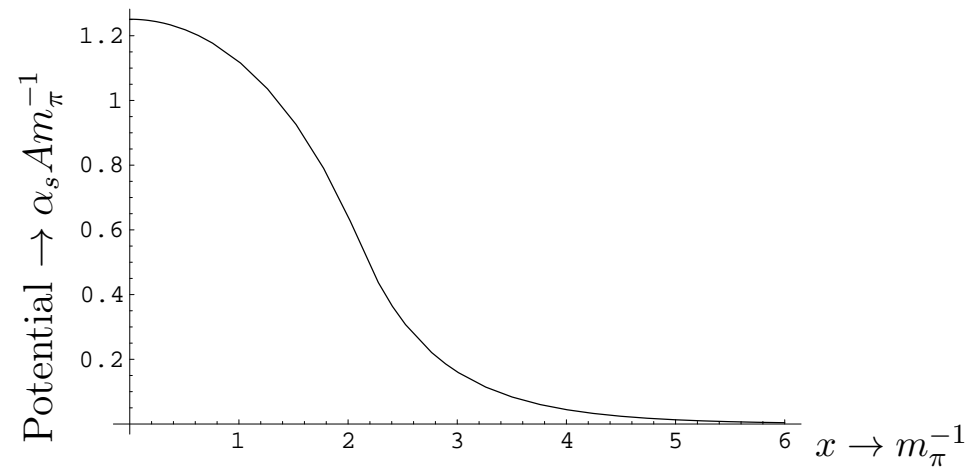

Fig. 6. The strong interaction (Yukawa like) potential felt by the elastically scattered neutrons for the $A=20$ system.

lower than the DM event rate of the present concern.

Neutrons in the $10 \mathrm{MeV}-1 \mathrm{GeV}$ region are of the order of 1 per $m^{2}$ per year at underground laboratories of $4000 m$ water 


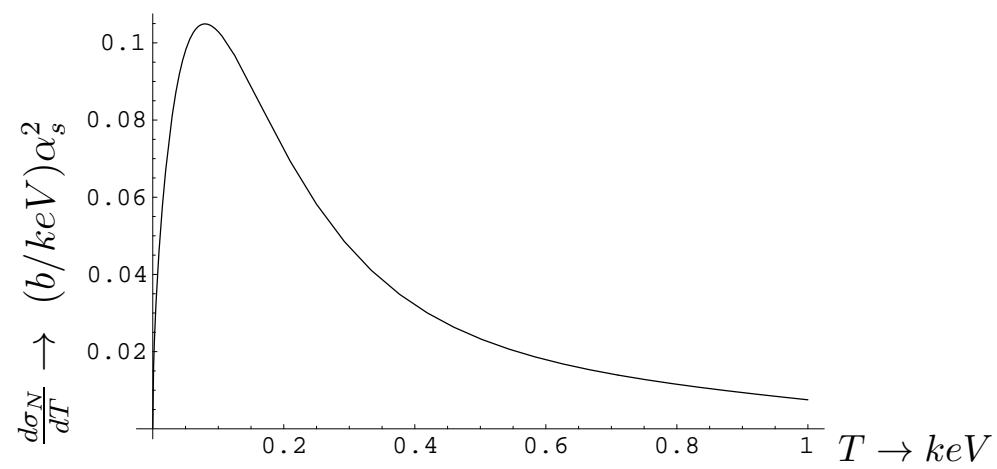

Fig. 7. The differential cross section for neutron electron scattering due to the strong interaction. The value $\alpha_{s}=1.0$ was used in the numerical calculation. Otherwise the notation is the same as in Fig. 4.

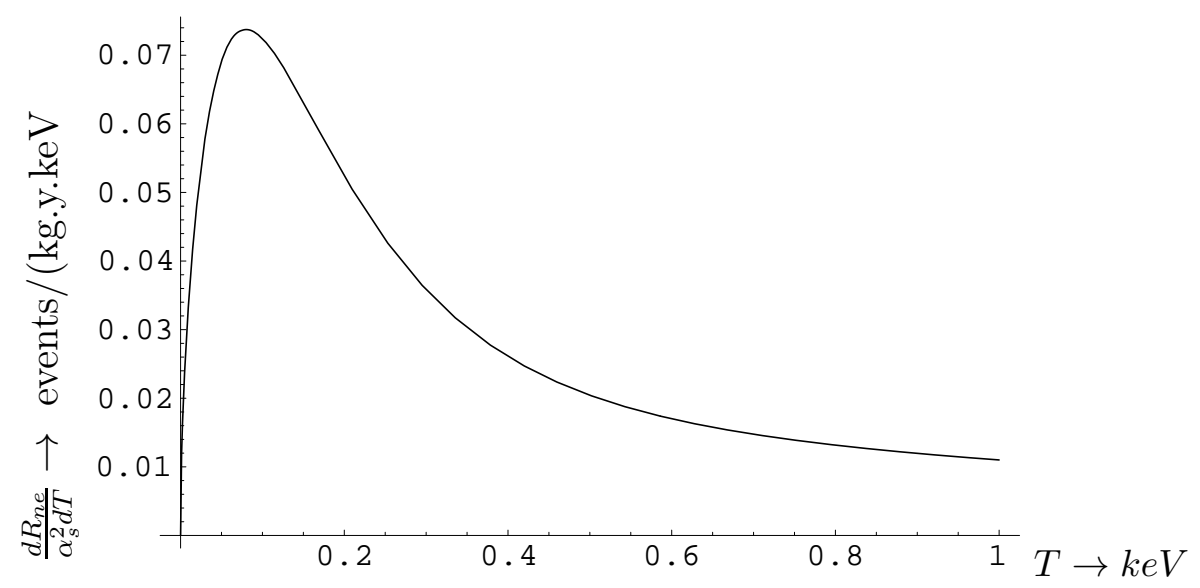

Fig. 8. The differential rate for electron ionization in elastic neutron nucleus scattering. Otherwise the notation is the same as in Fig. 5

equivalent. Lower energy neutrons arising from nuclear fission of Ur-Th isotopes around the detector can be slowed down and be absorbed.

This source is also present in the standard nuclear recoil experiments. Such neutron events can be deciphered by studying the reaction products. We will, however, estimate the event rate due to neutrons using the distribution for muon produced neutrons given by Gaitskell [31], approximated by:.

$$
f\left[e_{n}\right]=\left(1.72 e^{-\left[e_{n} /(241 \mathrm{MeV})\right]}+8.27 e^{-\left[e_{n} /(55.5 \mathrm{MeV})\right]}\right) / \mathrm{MeV} \cdot y \cdot m^{2} .
$$

We distinguish the following cases:

- Neutron electron scattering due to the neutron magnetic dipole 
moment. The leading term in this interaction is of the form:

$$
\Omega(\mathbf{r})=\sqrt{4 \pi \alpha} \frac{\mu_{n}}{2 m_{e}}\left[\sigma_{e} \times \sigma_{n}\right]^{2} \cdot \sqrt{4 \pi} Y^{2}(\hat{r}) \frac{1}{r^{3}} .
$$

Thus one must evaluate the orbital matrix element

$$
\int d^{3} \mathbf{x} \Omega(\sqrt{2} \mathbf{x}) e^{i \mathbf{k} \cdot \mathbf{x} \sqrt{2}} \int d^{3} \mathbf{y} \phi_{b}\left(\frac{\mathbf{x}-\mathbf{y}}{\sqrt{2}}\right),
$$

where $\phi_{b}$ is the bound electron wave function and $\mathbf{k}$ is the momentum of the outgoing electron. The evaluation of the above integral proceeds by a multipole expansion. For bound electrons in s-states the only multipole appearing is the quadrupole, while for p-states one can have both a dipole and octapole term with the dipole dominating. One can then compute the spin matrix elements and calculate the differential cross section as a function of the outgoing electron energy. One should use a proper neutron energy distribution function. For orientation purposes we calculated the differential cross section for a neutron energy of $\epsilon_{n}=2 \mathrm{keV}$, which leads to a maximum electron energy $\epsilon_{n}+\epsilon_{n \ell}$. The obtained results are shown in Fig. 4 . We see that the shape of the differential cross section is very different from that of the interesting electrons. Furthermore such events can be rejected by the subsequent detection of the neutron. The event rate, obtained by using the Gaitskell neutron distribution, is given in Fig. 5 .

- Neutron nucleus elastic scattering. This can lead to electron ejection in pretty much the same way as the LSP-Nucleus elastic scattering. Background events can be rejected by the detection of the scattere neutron.

Proceeding in a manner analogous to that described in sec. 2 we find:

$$
\begin{aligned}
& d \sigma(\mathbf{k})=\frac{1}{v} \frac{m_{e}}{E_{e}}|M|^{2} \frac{d^{3} \mathbf{p}_{n}^{\prime}}{(2 \pi)^{3}} \frac{d^{3} \mathbf{k}}{(2 \pi)^{3}}(2 \pi)^{3} \frac{1}{2(2 \ell+1)} \\
& \sum_{n \ell m} p_{n \ell} \quad\left[\tilde{\phi}_{n \ell m}(\mathbf{k})\right]^{2} 2 \pi \delta\left(\epsilon_{n}+\epsilon_{n \ell}-T-\epsilon_{n}^{\prime}-\frac{\left(\mathbf{p}_{n}-\mathbf{k}-\mathbf{p}_{n}^{\prime}\right)^{2}}{2 m_{A}}\right),
\end{aligned}
$$

with the amplitude $\mathrm{M}$ being just the Fourier transform of the 
potential generated by the A nucleons, which is felt by the neutrons:

$$
M=\int d^{3} \mathbf{r} e^{i\left(\mathbf{p}_{n}^{\prime}-\mathbf{p}_{n}\right) \cdot \mathbf{r}} V_{A}(r)=\tilde{V}_{A}\left(\left|\mathbf{p}_{n}^{\prime}-\mathbf{p}_{n}\right|\right) .
$$

Ignoring the energy taken away by the nucleus we finally find:

$$
\begin{aligned}
& d \sigma_{(n,(e, n))}\left(\epsilon_{n}, T\right)=\frac{1}{\pi \sqrt{2}} m_{n}^{2} m_{e} \sqrt{m_{e} T} d T \\
& \sum_{n \ell} p_{n \ell}\left|\tilde{\phi_{n \ell}}\left(\sqrt{2 m_{e} T}\right)\right|^{2} F_{A}\left(\epsilon, \frac{\epsilon_{n \ell}-T}{\epsilon_{n}}\right),
\end{aligned}
$$

with $F_{A}\left(\epsilon_{n}, x\right)$ given by:

$$
F_{A}\left(\epsilon_{n}, x\right)=\int_{1-\sqrt{1-x}}^{1+\sqrt{1-x}} t d t\left[\tilde{V}_{A}\left(b \sqrt{2 m_{n} \epsilon_{n}} t\right)\right]^{2},
$$

where $b$ is a length parameter characterizing the range of the potential.

The essential input in this case is the neutron-nucleus elastic scattering potential $V_{A}$, which can be determined phenomenologically.

Proceeding further the elastic neutron nucleus cross-section can be obtained in an analogous fashion:

$$
d \sigma_{(n, n)}\left(\epsilon_{n}\right)=\frac{1}{2 \pi} \frac{m_{n}}{2 \epsilon_{n}} \int_{0}^{\epsilon_{n}}\left(\epsilon_{n}-x\right) d x\left[\tilde{V}_{A}\left(b\left(\epsilon_{n}-x\right)\right)\right]^{2} .
$$

In other words the evaluation of the interesting cross-section requires a complicated analysis of the elastic neutron nucleus cross-section. Thus for the purposes of the present work we have decided to obtain the potential by folding the Yukawa interaction with a range $b=m_{\pi}^{-1}$ with a constant nuclear density . The thus obtained potential is shown in 6 . The obtained elastic neutron nucleus rate is presented in Fig. 8. We see that this background contribution is peaked at very low electron energy.

- Compound nucleus formation by neutron capture. Such background events can be rejected by detecting the decay $\gamma$-rays of the compound nucleus.

In this case, if one ignores the tiny energy taken away by the outgoing nucleus, for a neutron of energy $\epsilon_{n}$ the electron 
energy is fixed, $T=\epsilon_{n}+\epsilon_{n \ell}+\Delta-E_{x}$, where $\Delta$ is the available energy and $E_{x}$ is the energy of the of the compound nuclear state. For thermal neutrons the electron energy is essentially fixed by the energy of the compound nucleus $T \approx \epsilon_{n \ell}+\Delta-E_{x}$. Thus this source does not pose a serious problem.

Anyway, if one wants, one can cast the relevant cross section in the form:

$$
\begin{aligned}
\sigma\left(\epsilon_{n}\right)= & \frac{m_{e}}{2(2 \pi)^{2}} \sqrt{\frac{m_{n}}{2 \epsilon_{n}}} \sum_{n \ell, x} p_{n \ell} \sqrt{2 m_{e}\left(\epsilon_{n}+\epsilon_{n \ell}+\Delta-E_{x}\right)} \\
& \left|\tilde{\phi_{n \ell}}\left(\sqrt{2 m_{e}\left(\epsilon_{n}+\epsilon_{n \ell}+\Delta-E_{x}\right)}\right)\right|^{2}\left|M\left(p_{n}, x\right)\right|^{2},
\end{aligned}
$$

where $M\left(p_{n}, x\right)$ is the amplitude associated with the standard compound nucleus formation. Noting that the captured neutrons are thermal one finds that the event rate for this process can be related to the rate for neutron capture as follows:

$$
\begin{gathered}
\left.\frac{d N}{d t} \approx \frac{1}{2(2 \pi)^{3}} \int_{0}^{\epsilon} d \epsilon_{n} m_{e} \sum_{n \ell, x} p_{n \ell} \sqrt{2 m_{e}\left(\epsilon_{n}+\epsilon_{n \ell}+\Delta-E_{(A)}\right.} .10\right) \\
\left|\tilde{\phi_{n \ell}}\left(\sqrt{2 m_{e}\left(\epsilon_{n}+\epsilon_{n \ell}+\Delta-E_{x}\right)}\right)\right|^{2}\left(\frac{d N}{d t}\right)_{\text {compound }},
\end{gathered}
$$

where the last term in the above equation gives the rate for the compound nucleus formation and $\epsilon$ is the highest energy at which neutron capture can occur. We are not, however, going to elaborate further on this point.

The background rates have so far been evaluated by using the neutron flux at $2000 \mathrm{~m}$ w.e. given by Gaitskell. The BG rates are of the same order of magnitude as the true DM rates or smaller and are well reduced by requiring anti-coincidence with the reaction products. Furthermore present underground laboratories are typically at around $3000 \mathrm{~m}$ w.e. or deeper. Thus the neutron flux and the background rates at these depths get smaller by orders of magnitude compared to those at $2000 \mathrm{~m}$ w.e. It thus appears that the BG rates from neutrons are not serious. 


\section{References}

[1] S. Hanary et al, Astrophys. J. 545, L5 (2000);

J.H.P Wu et al, Phys. Rev. Lett. 87, 251303 (2001);

M.G. Santos et al, Phys. Rev. Lett. 88, 241302 (2002)

[2] P.D. Mauskopf et al, Astrophys. J. 536, L59 (20002);

S. Mosi et al, Prog. Nuc.Part. Phys. 48, 243 (2002);

S.B. Ruhl al, astro-ph/0212229 and references therein.

[3] N.W. Halverson et al, Astrophys. J. 568, 38 (2002)

L.S. Sievers et al astro-ph/0205287 and references therein.

[4] G.F. Smoot et al, (COBE data), Astrophys. J. 396, (1992) L1.

[5] D.N. Spergel et al, astro-ph/0302209

[6] A.H. Jaffe et al.,Phys. Rev. Lett. 86, 3475 (2001).

[7] M. Tegmark et al, Cosmological parameters from SDSS and WMAP, astro-ph/0310723

[8] D.P. Bennett et al., (MACHO collaboration), A binary lensing event toward the LMC: Observations and Dark Matter Implications, Proc. 5th Annual Maryland Conference, edited by S. Holt (1995);

C. Alcock et al., (MACHO collaboration), Phys. Rev. Lett. 74, 2967 (1995).

[9] R. Bernabei et al., INFN/AE-98/34, (1998); R. Bernabei et al., it Phys. Lett. B 389, 757 (1996) .

[10] R. Bernabei et al., Phys. Lett. B 424, 195 (1998); B 450, 448 (1999).

[11] A. Benoit et al, [EDELWEISS collaboration], Phys. Lett. B 545, 43 (2002); V. Sanglar,[EDELWEISS collaboration], arXiv:astro-ph/0306233

[12] D.S. Akerib et al,[CDMS Collaboration], Phys. Rev D 68, 082002 (2003) arXiv:astro-ph/0405033.

[13] For a review see:

G. Jungman, M. Kamionkowski and K. Griest, Phys. Rep. 267, 195 (1996).

[14] M.W. Goodman and E. Witten, Phys. Rev. D 31, 3059 (1985).

[15] K. Griest, Phys. Rev. Lett 61, 666 (1988).

[16] J. Ellis, and R.A. Flores, Phys. Lett. B 263, 259 (1991); Phys. Lett. B 300, 175 (1993); Nucl. Phys. B 400, 25 (1993).

[17] J. Ellis and L. Roszkowski, Phys. Lett. B 283, 252 (1992).

[18] For more references see e.g. our previous report:

J.D. Vergados, Supersymmetric Dark Matter Detection- The Directional Rate and the Modulation Effect, hep-ph/0010151. 
[19] M.E.Gómez and J.D. Vergados, Phys. Lett. B 512 , 252 (2001); hep-ph/0012020. M.E. Gómez, G. Lazarides and C. Pallis, Phys. Rev. D 61, 123512 (2000) and Phys. Lett. B 487, 313 (2000).

[20] M.E. Gómez and J.D. Vergados, hep-ph/0105115.

[21] A. Bottino et al., Phys. Lett B 402, 113 (1997). R. Arnowitt. and P. Nath, Phys. Rev. Lett. 74, 4952 (1995); Phys. Rev. D 54, 2394 (1996); hep-ph/9902237;

V.A. Bednyakov, H.V. Klapdor-Kleingrothaus and S.G. Kovalenko, Phys. Lett. B 329, 5 (1994).

[22] U. Chattopasdhyay A. Corsetti and P. Nath, Phys. Rev. D 68, 035005 (2003).

[23] U. Chattopasdhyay and D.P. Roy, Phys. Rev D 68, 033010 (2003); hep-ph/0304108.

[24] B. Murakami and J.D. Wells, Phys. Rev. D 64 (2001) 015001 hep-ph/0011082

[25] D.N. Spergel, Phys. Rev. 37, (1988) 1353

J.D. Lewin and P.F. Smith, Astropart. Phys. 6, (1996) 87

C.J. Copi, J. Heo and L.M. Krauss, Phys. Lett. 461 B , 43 (1999);

D. Akimov et al, Phys. Lett. 525 B, 245 (2002).

B. Morgan, A.M. Green and N. Spooner astro-ph/0408047.

[26] D.P. Snowden-Ifft, C.C. Martoff and J.M. Burwell, Phys. Rev. D 61. 1 (2000).; M. Robinson et al. Nucl. Inst. Meth. A 511, 347 (2003).

K.N. Buckland, M.J. Lehner and G,E, Masek, In Proc. 3nd Int. Conf. on Dark Matter in Astro and Part. Phys. (Dark2000), Ed. V.N. Klapdor-Kleingrothaus, Springer Verlag (2002).

[27] J.D. Vergados, Phys. Rev. D 67 (2003) 103003; ibid 58 (1998) 10301-1;

J.D. Vergados, J. Phys. G: Nucl. Part. Phys. 30, 1127 (2004).

[28] J.D. Vergados, P.Quentin and D. Strottman, hep-ph/0310365 (to be published)

[29] H. Ejiri, K. Fushimi and H. Ohsumi, Phys. Lett. B 317, 14 (1993).

[30] Y. Giomataris and J.D. Vergados, Nucl.Instr. Meth. A 530, 330 (2004).

[31] R. Gaitskell, astro-ph/0106200.

[32] G.J. Gounaris,E.A. Paschos and P.I. Porfyriadis, Phys. Lett. B 525 (2002) 63; hep-ph/0109183.

[33] P.C. Divari, T.S. Kosmas, J.D. Vergados and L.D. Skouras, Phys. Rev. C 61 (2000), 044612-1. 